\title{
A Phase II Trial of Everolimus in Patients with Advanced Pancreatic Neuroendocrine Carcinoma Refractory or Intolerant to Platinum-Containing Chemotherapy (NECTOR Trial)
}

\author{
Hiroyuki Okuyama ${ }^{a, b}$ Masafumi Ikedab Takuji Okusakac Masayuki Furukawad \\ Shinichi Ohkawa ${ }^{e}$ Ayumu Hosokawa ${ }^{f}$ Yasushi Kojimag Hiroki Harah \\ Gou Murohisai Kazuhiko Shiojij Akinori Asagik Nobumasa Mizuno' \\ Motohiro Kojimam $^{\mathrm{m}}$ Takeharu Yamanakan $^{\mathrm{n}}$ Junji Furuse ${ }^{\circ}$ \\ ${ }^{a}$ Department of Clinical Oncology, Faculty of Medicine, Kagawa University, Kagawa, Japan; ${ }^{b}$ Department of \\ Hepatobiliary and Pancreatic Oncology, National Cancer Center Hospital East, Kashiwa, Japan; ' Department of \\ Hepatobiliary and Pancreatic Oncology, National Cancer Center Hospital, Tokyo, Japan; d Department of \\ Hepato-Biliary-Pancreatology, National Hospital Organization Kyushu Cancer Center, Fukuoka, Japan; ${ }^{\mathrm{e} D i v i s i o n}$ of \\ Hepatobiliary and Pancreatic Oncology, Department of Gastroenterology, Kanagawa Cancer Center, Yokohama, \\ Japan; fDepartment of Gastroenterology and Hematology, Faculty of Medicine University of Toyama, Toyama, \\ Japan; ${ }^{9}$ Department of Gastroenterology, National Center for Global Health and Medicine, Tokyo, Japan; \\ ${ }^{h}$ Department of Gastroenterology, Saitama Cancer Center, Saitama, Japan; 'Department of Gastroenterology, \\ Seirei Hamamatsu General Hospital, Hamamatsu, Japan; 'Department of Internal Medicine, Niigata Cancer Center \\ Hospital, Niigata, Japan; ' Department of Gastrointestinal Medical Oncology, National Hospital Organization Shikoku \\ Cancer Center, Matsuyama, Japan; 'Department of Gastroenterology, Aichi Cancer Center Hospital, Nagoya, Japan; \\ mDivision of Pathology, Exploratory Oncology Research and Clinical Trial Center, National Cancer Center, Kashiwa,

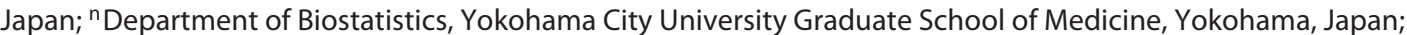 \\ ${ }^{\circ}$ Department of Medical Oncology, Kyorin University Faculty of Medicine, Tokyo, Japan
}

\section{Keywords}

Pancreatic neoplasms · Neuroendocrine carcinoma .

Everolimus · Platinum-containing chemotherapy

\begin{abstract}
Background: Platinum-containing regimens are widely used as first-line chemotherapy for unresectable pancreatic neuroendocrine carcinoma (NEC), but second-line chemotherapies have yet to be established. Objectives: We evaluated the safety and efficacy of everolimus in patients with
\end{abstract}

karger@karger.com www.karger.com/nen

Karger $\stackrel{\text { ' }}{5}$

BOPEN ACCESS
(C) 2020 The Author(s)

Published by S. Karger AG, Basel

This article is licensed under the Creative Commons AttributionNonCommercial-NoDerivatives 4.0 International License (CC BY NC-ND) (http://www.karger.com/Services/OpenAccessLicense) Usage and distribution for commercial purposes as well as any distribution of modified material requires written permission. pancreatic NEC refractory or intolerant to platinum-containing chemotherapy. Methods: This study was a prospective, multicenter, phase II trial in patients with pancreatic NEC after platinum-containing chemotherapy. Everolimus treatment was continued until disease progression or intolerable toxicity was observed. The primary endpoint was progression-free survival (PFS). Results: Participants comprised 25 patients. Median age was 63 years, median PFS was 1.2 months (95\% confidence interval $[\mathrm{Cl}]$ 0.9-3.1 months), median overall survival was 7.5 months $(95 \%$ Cl $3.1-13.5$ months), overall response rate was $0 \%$, and disease control 
rate was $39.1 \%$. Common grade $3 / 4$ adverse events were hyperglycemia (20\%), thrombocytopenia (16\%), and anemia (16\%). Conclusion: The efficacy of everolimus was limited in patients with unresectable pancreatic NEC.

(c) 2020 The Author(s)

Published by S. Karger AG, Basel

\section{Introduction}

Pancreatic neuroendocrine carcinoma (NEC) is an extremely rare tumor, reportedly accounting for $10 \%$ of pancreatic neuroendocrine neoplasms (NENs) [1]. If the tumors cannot be resected surgically, systemic chemotherapy is often administered. Although no large-scale, randomized, controlled trials have examined the survival benefit of chemotherapy, platinum-containing regimens are widely used as first-line standard chemotherapy [25]. However, no second-line chemotherapy has been established.

Everolimus is a molecularly targeted agent that inhibits mammalian target of rapamycin (mTOR). In the RADIANT-3 phase III trial for pancreatic neuroendocrine tumor (NET), progression-free survival (PFS) was longer with everolimus than with placebo [6]. Based on this result, everolimus has been acknowledged as one of the standard therapies for patients with unresectable pancreatic NET. In a subgroup analysis, patients with moderately differentiated NET showed a lower hazard ratio for PFS than patients with well-differentiated NET [6]. Moreover, patients with poorly differentiated NETs have shown higher rates of $\mathrm{p}$-mTOR expression in tumors compared to patients with well-differentiated tumors [7, 8]. In addition, good response to everolimus was obtained in a pancreatic NEC patient with a high expression of Ki$67(30-40 \%)$ [9]. Such results suggest that everolimus may be effective against poorly differentiated tumors. The present phase II trial was thus conducted to examine the efficacy and safety of everolimus in patients with advanced pancreatic NEC.

\section{Materials and Methods}

This study was a single-arm, phase II trial conducted at 12 institutions in Japan.

\section{Eligibility Criteria}

Eligibility criteria were as follows: age $\geq 20$ years; unresectable disease; histologically confirmed pancreatic NEC (according to the 2010 classification of the World Health Organization [WHO]); refractory or intolerant to platinum-containing chemotherapy; Eastern Cooperative Oncology Group performance status (ECOG PS)
$0-2$; at least 1 lesion of the disease evaluable on computed tomography; $\geq 3$ weeks since previous therapy; adequate bone marrow (neutrophil count $\geq 1,500 / \mu \mathrm{L}$, hemoglobin $\geq 8.5 \mathrm{~g} / \mathrm{dL}$, platelet count $\geq 75,000 / \mu \mathrm{L}$ ); adequate hepatic function (aspartate and alanine aminotransferase levels $\leq 2.5 \times$ upper limit of normal [ULN]); $\leq 5 \times$ ULN in patients with liver metastasis; total bilirubin level $\leq 2 \times$ ULN; adequate renal function (serum creatinine level $\leq 1.5 \mathrm{mg}$ / $\mathrm{dL}$ ), and written informed consent. Exclusion criteria were as follows: patients with NET G1 or G2; presence of interstitial pneumonitis; brain metastases; active infection; active gastroduodenal ulceration; other severe uncontrolled comorbidities such as diabetes, heart disease, mental disorder, or prior or concurrent malignancy with the preceding 3 years; using steroid or immunosuppressive agent, women during pregnancy or nursing; and prior therapy with mTOR inhibitors.

\section{Study Treatment}

Everolimus treatment was initiated orally at a dose of $10 \mathrm{mg}$ once daily and continued until disease progression or intolerable toxicity was observed. Dose reduction or delay was permitted according to the protocol when severe toxicity was seen. In such cases, 2 levels of dose reduction were permitted for everolimus: an initial reduction to $5 \mathrm{mg}$ /day (Level -1 ), or subsequent reduction to $5 \mathrm{mg}$ every other day (Level -2).

Efficacy and Safety Assessment

Clinical and laboratory evaluations were performed at least every 2 weeks. Adverse events were evaluated according to the Common Terminology Criteria for Adverse Events (CTCAE) version 4.0. Radiological response was evaluated by contrast-enhanced computed tomography performed every 1 month for the first 3 months, then every 2 months after 4 months. Tumor response was evaluated according to the Response Evaluation Criteria in Solid Tumors (RECIST), version 1.1 [10], to judge best overall response. In this trial, central pathological review was performed by an expert pathologist.

\section{Statistical Analysis}

The primary endpoint was PFS, defined as the time from the start of treatment to progression or death from any cause. Secondary endpoints were overall survival (OS; time from the start of treatment until the date of death), time to treatment failure (time from the start of treatment to discontinuation of everolimus or death from any cause), response rate (RR) (complete response [CR] or partial response [PR] according to RECIST version 1.1), disease control rate (DCR; CR, PR, or stable disease according to RECIST version 1.1), and adverse events.

In this study, the threshold PFS rate at 3 months was 25\% (corresponding to a median PFS of 1.5 months), and the expected PFS rate at 3 months was $50 \%$ (corresponding to a median PFS of 3.0 months). If the lower limit of the $90 \%$ confidence interval (CI) for the PFS rate at 3 months exceeded the threshold PFS rate (25\%), this treatment was considered promising. The planned sample size was 19 patients for $80 \%$ power and an alpha value of 0.05 (one sided). Kaplan-Meier methodology was used to estimate PFS and OS. The final analysis was performed 1 year after the last patient was enrolled in this trial. Analysis of predictors for PFS was performed using the Kaplan-Meier method, and differences were evaluated by the log-rank test. All analyses were performed using JMP for Windows version 10 software (SAS Institute, Cary, NC, USA). 


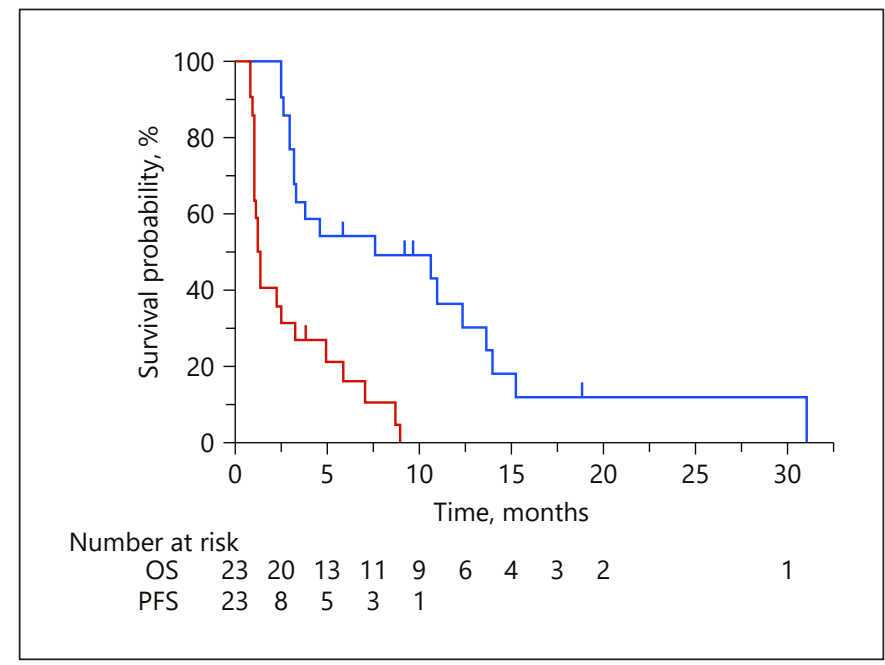

Fig. 1. Kaplan-Meier curves for progression-free survival (PFS) and overall survival (OS; $n=23$ ). The PFS rate at 3 months was $31.8 \%$ (90\% CI 18.0-49.8\%), and the median PFS was 1.2 months (95\% CI 0.9-3.1 months). The PFS rate at 1 month was $59.1 \%$ (95\% CI 41.4-74.7\%). Median OS was 7.5 months (95\% CI 3.1-13.5 months). Red line, PFS; blue line, OS.

\section{Results}

\section{Patient Characteristics}

Between February 2014 and March 2017, a total of 25 patients from 12 institutions were enrolled. All patients received everolimus therapy, but 2 patients were identified as ineligible after central pathological review yielded a diagnosis other than NEC; one had NET G2, and the other had a mixed acinar-endocrine tumor. These patients were included in the safety analysis but were excluded from efficacy analysis. Characteristics of the 23 eligible patients are summarized in Table 1. Median age of the patients was 63 years (range 39-82 years), and all patients were ECOG PS 0 or 1 . Union for International Cancer Control (UICC) stage was III in 1 patient (4\%) and IV in 22 patients (96\%). The Ki-67 index was $<55 \%$ in 14 patients $(61 \%)$. The number of previous regimens was 1 in 15 patients (65\%), and $\geq 2$ in 8 patients (34\%).

\section{Efficacy}

For the 23 patients eligible for efficacy analysis, objective RR and DCR were $0 \%$ and $39.1 \%$, respectively. The PFS rate at 3 months was $31.8 \%$ (90\% CI 18.0-49.8\%), and median PFS was 1.2 months (95\% CI 0.9-3.1 months; Fig. 1). Median OS was 7.5 months (95\% CI 3.1-13.5 months; Fig. 1). No difference in PFS was seen between patients with a Ki-67 index $\geq 55 \%$ or $<55 \%(p=0.35)$. Fur-
Table 1. Patient characteristics $(n=23)$

\begin{tabular}{|c|c|}
\hline Age, median (range), years & $63(39-82)$ \\
\hline \multicolumn{2}{|l|}{ Sex, $n(\%)$} \\
\hline Male & $15(65)$ \\
\hline Female & $8(35)$ \\
\hline \multicolumn{2}{|l|}{ ECOG PS, $n(\%)$} \\
\hline 0 & $10(44)$ \\
\hline 1 & $13(56)$ \\
\hline \multicolumn{2}{|l|}{ Primary site of pancreas, $n(\%)$} \\
\hline Head & $13(56)$ \\
\hline Body or tail & $10(44)$ \\
\hline \multicolumn{2}{|l|}{ UICC stage, $n(\%)$} \\
\hline III & $1(4)$ \\
\hline IV & $22(96)$ \\
\hline \multicolumn{2}{|l|}{$\mathrm{Ki}-67$ index, $n(\%)$} \\
\hline$<55 \%$ & $14(61)$ \\
\hline$\geq 55 \%$ & $9(39)$ \\
\hline Serum NSE, median (range), ng/mL & $31.5(5.9-293.5)$ \\
\hline \multicolumn{2}{|l|}{ Metastatic sites, $n(\%)$} \\
\hline Liver & $14(61)$ \\
\hline Lymph node & $8(35)$ \\
\hline Adrenal gland & $3(13)$ \\
\hline Others & $3(13)$ \\
\hline \multicolumn{2}{|l|}{ Previous treatment, $n(\%)$} \\
\hline \multicolumn{2}{|l|}{ Number of regimens } \\
\hline 1 & $15(65)$ \\
\hline 2 & $7(30)$ \\
\hline 3 & $1(4)$ \\
\hline Resection & $3(13)$ \\
\hline \multicolumn{2}{|l|}{ Chemotherapy } \\
\hline Cisplatin + irinotecan & $13(57)$ \\
\hline Cisplatin + etoposide & $8(35)$ \\
\hline Irinotecan & $3(13)$ \\
\hline Amrubicin & $3(13)$ \\
\hline Carboplatin + etoposide & $2(9)$ \\
\hline Others & $3(13)$ \\
\hline
\end{tabular}

ECOG PS, Eastern Cooperative Oncology Group performance status; UICC, Union for International Cancer Control; NSE, neuron-specific enolase.

ther treatment after everolimus therapy was administered to 14 patients (61\%). These subsequent therapeutic regimens were as follows: amrubicin in 5 patients (36\%); irinotecan in 3 patients (21\%); streptozocin in 3 patients $(21 \%)$; S-1 in 2 patients (14\%); sunitinib in 2 patients (14\%); and gemcitabine + nab-paclitaxel in 2 patients (14\%).

\section{Safety}

Median duration of everolimus therapy was 1.2 months (range 0.1-8.8 months), and dose reductions of everolimus were performed for 5 patients. Adverse events are summarized in Table 2. The most common grade 3 or 4 
Table 2. Adverse events in $\geq 20 \%$ of patients $(n=25$ )

\begin{tabular}{lll}
\hline Adverse event & All grades, $n(\%)$ & Grade 3 or $4, n(\%)$ \\
\hline Anemia & $24(96)$ & $4(16)$ \\
Hypoalbuminemia & $20(80)$ & 0 \\
Thrombocytopenia & $18(72)$ & $4(16)$ \\
Hyponatremia & $14(56)$ & $3(12)$ \\
Fatigue & $14(56)$ & $2(8)$ \\
Anorexia & $13(52)$ & $2(8)$ \\
Stomatitis & $12(48)$ & $1(4)$ \\
Neutropenia & $11(44)$ & $1(4)$ \\
Leukopenia & $10(40)$ & 0 \\
Elevated creatinine & $8(32)$ & 0 \\
Hypokalemia & $5(20)$ & $1(4)$ \\
Hyperkalemia & $5(20)$ & 0 \\
Diarrhea & $5(20)$ & 0 \\
Hyperglycemia & - & $5(20)$ \\
\hline
\end{tabular}

adverse events were hyperglycemia (20\%), anemia (16\%), and thrombocytopenia (16\%). Grade 4 rhabdomyolysis occurred in 1 patient, but the patient recovered with suspension of everolimus and sufficient fluid infusion. One patient discontinued study treatment because of rhabdomyolysis, but no other patients discontinued everolimus therapy due to adverse events. Twenty patients died, and no patients were receiving everolimus treatment at the time of final analysis.

\section{Discussion/Conclusion}

No large-scale trials have examined the survival benefit of first-line chemotherapy among patients with pancreatic NEC, but platinum-containing regimens are recommended as the de facto standard first-line chemotherapy $[11,12]$. However, no second-line chemotherapies have been established. This was the first prospective, multicenter, phase II study to elucidate the efficacy and safety of everolimus for pancreatic NEC. However, since the lower limit of the $90 \%$ CI of the PFS rate at 3 months was below the threshold PFS rate (25\%), the primary endpoint was not met in this trial. Further, median PFS was 1.2 months, median OS was 7.5 months, and DCR was $39.1 \%$, indicating a disappointing efficacy of everolimus. Median PFS and OS with sunitinib monotherapy were reported as 42 and 146 days in patients with NET G3/NEC, respectively [13], and those results were comparable to those of the present trial. With regard to cytotoxic chemotherapy, median PFS has been reported in the range of 2.1-6 months [14-16]. As to peptide receptor radionuclide therapy, median PFS was 14 months [17]. From these results, the efficacy of second-line chemotherapy is limited in patients with advanced NET G3/NEC. NET G3/NEC are aggressive tumors compared with NET G1 and G2, so the efficacy of monotherapy with a molecularly targeted agent was limited. More promising regimens, such as the combination of a molecularly targeted agent and a cytotoxic agent, should be considered for pancreatic NEC.

The present trial found no significant difference in PFS between Ki-67 $<55$ and $\geq 55 \%$. The efficacy of everolimus was not compared between NET G3 and NEC using the WHO 2019 classification in the present study. However, the efficacy of everolimus monotherapy appeared limited in patients with NET G3, as well as NEC, based on the short PFS for both high and low Ki-67 indices. In the present study, 2 patients achieved PFS $\geq 5$ months. For bronchial NETs, mTOR, p70S6K, AKT, and ERK1/2 proteins have been reported as potentially showing some utility in predicting resistance to $\mathrm{mTOR}$ and $\mathrm{PI} 3 \mathrm{~K} / \mathrm{mTOR}$ inhibitors [18]. We had hoped to examine biomarkers for the efficacy of everolimus but were unable to obtain sufficient tissues to examine these issues.

In terms of adverse events, the profile differed from that in RADIANT-3 trial. In the RADIANT-3 trial, common adverse events were stomatitis (64\%), rash (49\%), and diarrhea (34\%). In the present trial, these adverse events tended to be less frequent than in the RADIANT-3 trial (stomatitis, 48\%; rash, 12\%; diarrhea, 20\%). However, anemia, hypoalbuminemia, thrombocytopenia, hyponatremia, and fatigue were common adverse events. These adverse events were likely caused by tumor progression. Moreover, few patients discontinued treatment due to adverse events. Everolimus monotherapy appears to have been well tolerated.

Some limitations to this trial must be considered when interpreting the results. This was a single-arm trial with limited sample size. Currently, pancreatic NEN is classified according to the WHO 2019 classification, whereas the present study applied the WHO 2010 classification. Patients with NET G3 and NEC were thus included in this trial, and NET G3 could not be distinguished from NEC.

In conclusion, the efficacy of everolimus monotherapy was disappointing. More promising regimens need to be identified for pancreatic NEC.

\section{Acknowledgement}

We wish to thank all the patients and their families for participating in this study. We are grateful to the members of the National Cancer Center Exploratory Oncology Research and Clinical 
Trial Center for their support with data management (Mr. Yoshihiro Aoyagi, Ms. Seiko Matsuda), the members of the NECTOR trial data center (Ms. Kayo Takei), the members of the study committee for safety and efficacy monitoring (Dr. Toshihiko Doi, Dr. Shigeru Mikami, and Dr. Satoru Iwasa), and all the investigators in this trial.

\section{Statement of Ethics}

This study was approved by the Institutional Review Boards at each participating institution and was conducted ethically in accordance with the World Medical Association Declaration of Helsinki. Each patient provided a written consent prior to enrolment.

\section{Disclosure Statement}

M.I. reports personal fees and research funding from Novartis, personal fees from Teijin Pharma, Nobelpharma, during the conduct of the study; personal fees and research funding from Bayer, Eisai, ASLAN, Yakult, Bristol-Myers Squibb, Eli Lilly, Sumitomo Dainippon, Chugai, Kyowa Hakko Kirin, NanoCarrier, Taiho, and personal fees from MSD, Abbott, EA Pharma, Daiichi Sankyo, Shire, Otsuka, Kaken Pharmaceutical, Gilead, Astellas Pharma, Micron, and research funding from J Pharma, Kowa, AstraZeneca, Baxalta, Merck Serono, Zeria, Takeda Pharmaceutical, outside the submitted work; T.O. reports grants and personal fees from Novartis Pharma K.K. during the conduct of the study; grants and personal fees from Eli Lilly, Eisai Co., Ltd., Dainippon Sumitomo Pharma Co., Ltd., Taiho Pharmaceutical Co., Ltd., Bristol-Myers Squibb Company, Ono Pharmaceutical Co., Ltd., Pfizer Jana Inc., Bayer Yakuhin, Ltd., Chugai Pharmaceutical Co., Ltd., Yakuruto Honsha Co., Ltd., AstraZeneca K.K., Zeria Pharmaceutical Co., Ltd., grants from Kowa K.K., Kyowa Hakko Kirin Co., Ltd., Baxter, Meiji Seika Pharma Co., Ltd., personal fees from Daiichi Sankyo Co., Ltd., Nippon Chemifa Co., Ltd., EA Pharma Co., Ltd., FUJIFILM RI Pharma Co., Ltd., Celgene, K.K., MSD, K.K., Teijin Pharma, Ltd., Shire, AbbVie, Takeda Pharmaceutical Co., Ltd., outside the submitted work; S.O. reports personal fees from Yakult Honsha, Otsuka, MSD, Pfizer, outside the submitted work; A.H. reports personal fees from Taiho Pharmaceutical, Takeda, Ono Pharmaceutical, Novartis, Eisai, Chugai Pharma, Lilly, Daiichi Sankyo, Teijin Pharma, Sanofi, Merck, research funding from Chugai Pharma, Taiho Pharmaceutical, Ono Pharmaceutical, Eisai, Yakult Honsha, outside the submitted work; Y.K. reports personal fees from Takeda Pharmaceutical, Taiho, Sanofi, Bristol-Myers Squibb, Eli Lilly, Ono, BeiGene, NanoCarrier, outside the submitted work; H.H. reports grants from the Japan Agency for Medical Research and Development (AMED), during the conduct of the study; grants and personal fees from Daiichi Sankyo, MSD, Taiho, Chugai, ONO, BMS, grant from Merck Serono, Eisai, LSK BioPharma, Incyte, Pfizer, BoehringerIngelheim, Beigene, personal fees from Lilly, Yakult Honsha, Sanofi, Takeda, outside the submitted work; A.A. reports personal fees from Teijin Phama, outside the submitted work; N.M. reports grants from the Ministry of Health, Labour and Welfare, Japan, grants, personal fees and other from Novartis, during the

conduct of the study; grants and personal fees from Yakult Honsha, Taiho Pharmaceutical, grants from Merck Serono, AstraZeneca, Zeria Pharmaceutical, NanoCarrier, Eisai, MSD, Dainippon Sumitomo Pharma, ASLAN Pharmaceuticals, Incyte Inc., Pharma Valley Center, outside the submitted work; T.Y. reports grants and personal fees from Takeda, Chugai, Boehringer Ingelheim, Taiho, Daiichi-Sankyo, Bayer, grants from Ono, Merck Serono, Astellas, Eli Lilly, personal fees from Pfizer, Sysmex, Huya Biosciences, Gilead Sciences, outside the submitted work; J.F. reports personal fees from Novartis, grants from the National Cancer Center, the Ministry of Health, Labour and Welfare of Japan, during the conduct of the study; grants from Ono Pharmaceutical, MSD, Yakult Honsha, Sumitomo Dainippon, AstraZeneca, Takeda, Daiichi Sankyo, Taiho Pharmaceutical, Chugai Pharma, Sanofi, Daiichi Sankyo, Astellas Pharma, NanoCarrier, Eli Lilly Japan, Bayer Yakuhin, Kyowa Hakko Kirin, Mochida Pharmaceutical, Pfizer, Eisai, personal fees from Eisai, Bayer Yakuhin, Taiho Pharmaceutical, Ono Pharmaceutical, Yakult Honsha, Teijin Pharma, Shionogi, EA Pharma, Eli Lilly Japan, Takeda, Chugai Pharma, Fujifilm, Mochida Pharmaceutical, Nihon Servier, Sanofi, Fujifilm Toyama Chemical, Nobel pharma, Pfizer, Sawai Pharmaceutical, Daiichi Sankyo, Sumitomo Dainippon, Astellas Pharma, Merck Serono, Nippon Kayaku, MSD, Shire, AstraZeneca, JPharma, AbbVie, Kyowa Hakko Kirin, Takara Bio, Otsuka, outside the submitted work. The remaining authors have no conflicts of interest to declare.

\section{Funding Sources}

This work was supported by the National Cancer Center Research and Development Fund (23-A-22); Health and Labour Sciences Research Grants for Clinical Cancer Research (H23-006) from the Ministry of Health, Labour and Welfare of Japan.

\section{Author Contributions}

M.I. and H.O. designed the study and wrote the initial draft of the manuscript. T.Y. contributed to analysis and interpretation of data. All other authors have contributed to data collection and interpretation, and critically reviewed the manuscript. All authors approved the manuscript to be published and agree to be accountable for all aspects of the work in ensuring that questions related to the accuracy or integrity of any part of the work are appropriately investigated and resolved.

References
1 Strosberg JR, Cheema A, Weber J, Han G, Coppola D, Kvols LK. Prognostic validity of a novel American Joint Committee on Cancer Staging Classification for pancreatic neuroendocrine tumors. J Clin Oncol. 2011 Aug; 29(22):3044-9.

2 Moertel CG, Kvols LK, O'Connell MJ, Rubin J. Treatment of neuroendocrine carcinomas with combined etoposide and cisplatin. Evidence of major therapeutic activity in the anaplastic variants of these neoplasms. Cancer. 1991 Jul;68(2):227-32. 
3 Mitry E, Baudin E, Ducreux M, Sabourin JC, Rufié P, Aparicio T, et al. Treatment of poorly differentiated neuroendocrine tumours with etoposide and cisplatin. Br J Cancer. 1999 Dec;81(8):1351-5.

4 Fjällskog ML, Granberg DP, Welin SL, Eriksson C, Oberg KE, Janson ET, et al. Treatment with cisplatin and etoposide in patients with neuroendocrine tumors. Cancer. 2001 Sep; 92(5):1101-7.

5 Plöckinger U, Rindi G, Arnold R, Eriksson B, Krenning EP, de Herder WW, et al.; European Neuroendocrine Tumour Society. Guidelines for the diagnosis and treatment of neuroendocrine gastrointestinal tumours. A consensus statement on behalf of the European Neuroendocrine Tumour Society (ENETS). Neuroendocrinology. 2004;80(6):394-424.

6 Yao JC, Shah MH, Ito T, Bohas CL, Wolin EM, Van Cutsem E, et al.; RAD001 in Advanced Neuroendocrine Tumors, Third Trial (RADIANT-3) Study Group. Everolimus for advanced pancreatic neuroendocrine tumors. N Engl J Med. 2011 Feb;364(6):514-23.

7 Shida T, Kishimoto T, Furuya M, Nikaido T, Koda K, Takano S, et al. Expression of an activated mammalian target of rapamycin (mTOR) in gastroenteropancreatic neuroendocrine tumors. Cancer Chemother Pharmacol. 2010 Apr;65(5):889-93.
8 Catena L, Bajetta E, Milione M, Ducceschi M, Valente M, Dominoni F, et al. Mammalian target of rapamycin expression in poorly differentiated endocrine carcinoma: clinical and therapeutic future challenges. Target Oncol. 2011 Jun;6(2):65-8.

9 Fonseca PJ, Uriol E, Galván JA, Alvarez C, Pérez Q, Villanueva N, et al. Prolonged clinical benefit of everolimus therapy in the management of high-grade pancreatic neuroendocrine carcinoma. Case Rep Oncol. 2013 Aug;6(2):441-9.

10 Eisenhauer EA, Therasse P, Bogaerts J, Schwartz LH, Sargent D, Ford R, et al. New response evaluation criteria in solid tumours: revised RECIST guideline (version 1.1). Eur J Cancer. 2009 Jan;45(2):228-47.

11 National Comprehensive Cancer Network (NCCN). Neuroendocrine Tumors 2018. Available from: http://www.nccn.org

12 Garcia-Carbonero R, Sorbye H, Baudin E, Raymond E, Wiedenmann B, Niederle B, et al.; Vienna Consensus Conference participants. ENETS Consensus Guidelines for High-Grade Gastroenteropancreatic Neuroendocrine Tumors and Neuroendocrine Carcinomas. Neuroendocrinology. 2016;103(2): 186-94.

13 Pellat A, Dreyer C, Couffignal C, Walter T, Lombard-Bohas C, Niccoli P, et al. Clinical and Biomarker Evaluations of Sunitinib in Patients with Grade 3 Digestive Neuroendocrine Neoplasms. Neuroendocrinology. 2018; 107(1):24-31.
14 Welin S, Sorbye H, Sebjornsen S, Knappskog S, Busch C, Oberg K. Clinical effect of temozolomide-based chemotherapy in poorly differentiated endocrine carcinoma after progression on first-line chemotherapy. Cancer. 2011 Oct;117(20):4617-22.

15 Olsen IH, Sørensen JB, Federspiel B, Kjaer A, Hansen CP, Knigge U, et al. Temozolomide as second or third line treatment of patients with neuroendocrine carcinomas. ScientificWorldJournal. 2012;2012:170496.

16 Olsen IH, Knigge U, Federspiel B, Hansen CP, Skov A, Kjær A, et al. Topotecan monotherapy in heavily pretreated patients with progressive advanced stage neuroendocrine carcinomas. J Cancer. 2014 Aug;5(8):628-32.

17 Carlsen EA, Fazio N, Granberg D, GrozinskyGlasberg S, Ahmadzadehfar H, Grana CM, et al. Peptide receptor radionuclide therapy in gastroenteropancreatic NEN G3: a multicenter cohort study. Endocr Relat Cancer. 2019 Feb;26(2):227-39.

18 Gagliano T, Bellio M, Gentilin E, Molè D, Tagliati F, Schiavon M, et al. mTOR, p70S6K, AKT, and ERK1/2 levels predict sensitivity to $\mathrm{mTOR}$ and PI3K/mTOR inhibitors in human bronchial carcinoids. Endocr Relat Cancer. 2013 Jun;20(4):463-75. 